\title{
GERMINAÇÃO DE SEMENTES DE FEIJOEIRO “OURO VERMELHO” SUBMETIDAS A DIFERENTES PERÍODOS DE EXPOSIÇÃO E CONCENTRAÇÕES DE GA3
}

\author{
PAULA, Luiz Miguel de ${ }^{1}$ \\ SOUZA-LEAL, Thiago de ${ }^{2}$ \\ MANTOAN, Paula ${ }^{1}$ \\ PEDROSO-DE-MORAES, Cristiano ${ }^{3}$
}

ISSUE DOI: $10.3738 / 1982.2278 .911$

\begin{abstract}
RESUMO: Apesar das giberelinas serem essenciais para a germinação, a sua aplicação em sementes de feijoeiro cv. Ouro Vermelho, ainda não foi descrita. Dessa forma, o presente trabalho apresentou por objetivo verificar a influência de diferentes períodos de exposição e concentrações de $\mathrm{GA}_{3}$ na germinação de Phaseolus vulgaris $\mathbf{L}$. cv. "Ouro Vermelho". As sementes foram submetidas à pré-embebição por 30, 60 e 120 minutos em água e em concentrações de 5,10 e $20 \mathrm{mg} . \mathrm{L}^{-1}$ de $\mathrm{GA}_{3}$. Posteriormente, foram distribuídas em lotes de quatro placas de petri previamente forradas com duas folhas de papel filtro umedecido com $10 \mathrm{~mL}$ de água destilada, contendo 25 sementes cada, e submetidas à temperatura de $25 \pm 2^{\circ} \mathrm{C}$, e luz branca de lâmpadas fluorescentes a $32,85 \mu \mathrm{mol} . \mathrm{m}^{2} . \mathrm{s}^{-1}$ ao nível da semente e mantidas até sua germinação. As interações entre as concentrações de $\mathrm{GA}_{3}$ e períodos de exposição foram negativos para Tempo Médio de Germinação e positivos para a Germinabilidade e Índice de Velocidade de Germinação, com estas últimas alcançando valores médios maiores à concentração de $20 \mathrm{mg} \cdot \mathrm{L}^{-1}$ sob períodos de exposição ao regulador vegetal de 30 e 120 minutos respectivamente.
\end{abstract}

Palavras-chave: Phaseolus vulgaris. Giberelina. Germinabilidade.

SUMMARY: Germination of beans "Red Gold" (Fabaceae) submitted to different periods and concentrations of gibberellic acid). Although the gibberellins are essential for germination, their application to seeds of bean cv. "Red Gold" plants have not been described. Thus, this study presented aimed to investigate the influence of different exposure times and concentrations of $\mathrm{GA}_{3}$ on germination of Phaseolus vulgaris L. cv. "Red Gold". The seeds were subjected to pre-soaking for 30, 60 and 120 minutes and in water at concentrations of 5,10 and $20 \mathrm{mg} . \mathrm{L}^{-1} \mathrm{GA}_{3}$. Later, they were distributed in batches of four petri dishes previously lined with two sheets of filter paper moistened with $10 \mathrm{~mL}$ of distilled water containing 25 seeds each, and subjected to a temperature of $25 \pm 2{ }^{\circ} \mathrm{C}$, and white light bulbs with fluorescent ones $32.85 \mu \mathrm{mol} . \mathrm{m}^{-2} . \mathrm{s}^{-1}$ level of the seed and maintained until germination. The interactions between the GA3 concentrations and exposure times were negative for Mean Time Germination and positive for Germinability and Germination Speed Index, with the latter reaching values higher average concentration of 20 $\mathrm{mg} . \mathrm{L}^{-1}$ under exposure periods the plant regulator 30 and 120 minutes respectively.

Keywords: Phaseolus vulgaris. Gibberellin. Germination.

\section{INTRODUÇÃO}

As sementes protegem o embrião e as reservas nutritivas fornecem alimento para o novo organismo em desenvolvimento (SWARNKAR; POONAM ; MAURYA, 2004; RAVEN; EVERT ; EICHHORN, 2007). A absorção de água pelas sementes é o primeiro passo da germinação, sem o qual este processo não pode ocorrer (TAIZ; ZEIGER, 2004). Contudo, durante o processo germinativo o ácido giberélico desempenha papel fundamental, pois, ativa a síntese de enzimas que irão hidrolisar as reservas seminais, fornecendo a energia necessária para o crescimento do embrião (KIGEL ; GALILI, 1995; SWARNKAR;

\footnotetext{
${ }^{1}$ Alunos de Iniciação Científica do Centro Universitário Hermínio Ometto - Uniararas. Av. Maximiliano Baruto, 500. CEP: 13706-900. Araras, SP. Brasil.

${ }^{2}$ Mestrando do Departamento de Botânica, IB-Unesp, Caixa Postal 199, 13506-900 Rio Claro, SP, Brasil.

${ }^{3}$ Docente do Centro Universitário Hermínio Ometto - Uniararas. Av. Maximiliano Baruto, 500. CEP: 13706-900. Araras, SP. Brasil. Pós-Doutorando do Departamento de Biologia. Universidade de Aveiro, CEP: 3810-193, Aveiro, AV, Portugal. E-mail: pedroso@uniararas.br
} 
POONAM ; MAURYA, 2004; TAIZ ; ZEIGER, 2004), além de estimular o alongamento celular, permitindo o desenvolvimento mais acentuado da radícula e da parte área e, consequentemente, uniformização do processo germinativo (SALISBURY ; ROSS,1992).

O feijão (Phaseolus vulgaris L.) é considerado o mais importante representante da família Fabaceae, principalmente por ser a espécie cultivada mais antiga e utilizada nos continentes (EMBRAPA, 2013). Apresenta-se como um dos mais importantes constituintes da dieta do brasileiro por seu alto valor nutricional e um dos produtos agrícolas de maior importância, sendo cultivando na maioria dos estados do país (EMBRAPA, 2013; VIEIRA; PAULA-JÚNIOR, ; BORÉM, 1998).

O feijoeiro apresenta fruto legume, deiscente, constituído de duas valvas unidas por duas suturas, uma dorsal e outra ventral. A cor varia de acordo com a cultivar, podendo ser uniforme ou apresentar estrias e variar de acordo com o grau de maturação, de verde até marrom (VIEIRA ; RAVIS, 2000). A semente é exalbuminosa, com alto teor de carboidratos e proteínas e é constituída externamente de um tegumento, hilo, micrópila e rafe, podendo apresentar diferentes tamanhos e ampla variedade de cores (VIEIRA ; RAVIS, 2000; EMBRAPA, 2013). Internamente apresenta embrião formado por uma plúmula, duas folhas primárias, hipocótilo, dois cotilédones e radícula. Pode apresentar diferentes tamanhos e ampla variedade de cores (VIEIRA ; RAVIS, 2000; EMBRAPA, 2013).

A escolha da cultivar (cv.) adequada está entre os fatores que mais afetam o sucesso em relação à cultura do feijoeiro. Os consumidores de feijão de Minas Gerais e regiões vizinhas têm diferentes preferências pela cor dos grãos. A predominância é pelos grãos tipo carioca, havendo áreas que consomem predominantemente feijão preto. Mais recentemente, os consumidores da Zona da Mata têm preferido os feijões vermelhos (RAMALHO, 2005). A cv. "Ouro Vermelho", por exemplo, apresenta boa aceitação por parte dos consumidores e maior adaptação às condições de cultivo da região norte de Minas Gerais, que apresenta como principais características edáficas maior salinidade e menor disponibilidade hídrica as plantas (RAMALHO, 2005; SOUZA et al., 2010; EMBRAPA, 2013). Ainda, tal cultivar apresenta caracteres fitotécnicos importantes, tais como: peso médio de 100 sementes de $25 \mathrm{~g}$, porte semi-ereto, ciclo precoce a normal e reação intermediária à mancha-angular e ferrugem (RAMALHO, 2005).

Para o feijoeiro, sabe-se que o uso de $\mathrm{GA}_{3}$ pode afetar o crescimento e a fisiologia da planta (SOUZA et al., 2009; SOUZA et al., 2010). Dentre os trabalhos na literatura, encontram-se relatos da aplicação de $\mathrm{GA}_{3}$ na parte aérea e seu efeito no padrão de alocação de carboidratos (MORRIS ; ARTHUR, 1985), e no decréscimo da capacidade da planta em tolerar a remoção de folhas devido aos estresses bióticos e abióticos (CIPOLLINI JR., 1997).

$\mathrm{O}$ uso de $\mathrm{GA}_{3}$ com o objetivo específico de acelerar e uniformizar a germinação de Phaseolus vulgaris L. cv. "Ouro Vermelho" é inexistente na literatura. Dessa forma, o presente trabalho apresenta por objetivo verificar a influência de diferentes concentrações e períodos de exposição de $\mathrm{GA}_{3}$ na germinação desta cultivar de feijão.

\section{MATERIAL E MÉTODOS}

O presente trabalho foi desenvolvido no Laboratório de Botânica e Análises Ambientais do Centro Universitário Hermínio Ometto - UNIARARAS.

As sementes de Phaseolus vulgaris L. cv. "Ouro Vermelho", recém-coletadas do jardim experimental do campus do Centro Universitário Hermínio Ometto - Uniararas, foram triadas quanto ao tamanho, e as maiores foram submetidas à pré-embebição por 30, 60 e 120 em água e em concentrações de 5, 10 e 20 mg...- de GA 
Grupos de 25 unidades foram distribuídos em quatro placas de Petri de $9 \mathrm{~cm}$ de diâmetro, previamente esterilizadas, forradas com papel de filtro e umedecidas com $10 \mathrm{~mL}$ de água destilada (CURIEL ; MORAES, 2011). As placas permaneceram em Câmara de Germinação B.O.D. (MA 403) até sua germinação, sob temperatura de $25 \pm 2{ }^{\circ} \mathrm{C}$, e luz branca de lâmpadas fluorescentes a $32,85 \mu \mathrm{mol} . \mathrm{m}^{2} . \mathrm{s}^{-}$ ${ }^{1}$ ao nível da semente (BATISTA et al., 2012; FERNANDES et al., 2012).

Diariamente as sementes foram contadas e àquelas para as quais houve ocorrência de protrusão radicular foram consideradas germinadas (CURIEL ; MORAES, 2011). Os dados obtidos foram utilizados para o cálculo da Germinabilidade (G\%), do Índice de Velocidade de Germinação (IVG) e do Tempo Médio de Germinação (TMG) expresso em dias (BATISTA et al., 2012; FERNANDES et al., 2012).

$\mathrm{O}$ experimento foi conduzido em esquema inteiramente casualizado ( 3 x 4 - três períodos de exposição x quatro concentrações de ácido giberélico). Para a análise estatística, foi aplicada a análise de variância (ANOVA), seguida pelo teste de Tukey a 5\% de significância, com o auxilio do aplicativo BioEstat 5 (AYRES et al., 2007).

\section{RESULTADOS E DISCUSSÃO}

Apesar das giberelinas serem essenciais para a germinação (SWARNKAR et al., 2004), para Phaseolus, existe apenas o relato de que tal regulador de crescimento apresentou-se essencial no desenvolvimento in vitro de plântulas oriundas de embriões imaturos destacados (SKENE, 1969; SOUZA et al., 2010). Mesmo existindo trabalhos com feijoeiro que apresentam resultados favoráveis em relação à aplicação de Stimulate ${ }^{\circledR}$, não se pode afirmar com certeza que somente a giberelina contida no produto é a responsável pelo altos índices de G\% e IVG encontrados, uma vez que em sua formulação também existem citocinas (CASTRO ; VIEIRA, 2001), as quais podem influenciar nestas variáveis, principalmente, em sementes que apresentam altos índices de proteínas, como os representantes do gênero Phaseolus (VIEIRA ; RAVIS, 2000; EMBRAPA, 2013).

Para este trabalho, em relação à variável analisada Germinabilidade $(\mathrm{G} \%)$, o melhor resultado foi o obtido para a concentração de $20 \mathrm{mg} \cdot \mathrm{L}^{-1}$ de $\mathrm{GA}_{3}$ à 30 minutos de exposição. Entretanto, para a variável, Índice de Velocidade de Germinação (IVG), o melhor resultado obtido foi para a concentração de 20 mg.L.' de $\mathrm{GA}_{3}$ à 120 minutos de exposição (Tabela 1).

Tabela 1 - Período de exposição e concentração de $\mathrm{GA}_{3}$ na Germinabilidade (G\%), Índice de Velocidade de Germinação (IVG) e Tempo Médio de Germinação (TMG) em sementes de Phaseolusvulgaris cv. "Ouro Vermelho".

\begin{tabular}{|c|c|c|c|c|}
\hline \multirow[b]{2}{*}{$\begin{array}{l}\text { Período } \\
\text { (minutos) }\end{array}$} & \multicolumn{4}{|c|}{ Concentrações de $\mathbf{G A}_{3}$} \\
\hline & $\mathbf{H}_{2} \mathbf{O}$ & 5 mg.L ${ }^{-1}$ & $10 \mathrm{mg} \cdot \mathrm{L}^{-1}$ & $20 \mathrm{mg} \cdot \mathrm{L}^{-1}$ \\
\hline & \multicolumn{4}{|c|}{ G\% } \\
\hline 30 & $78,25 \mathrm{aC}^{1}$ & $95,75 \mathrm{aB}$ & $95,75 \mathrm{aB}$ & $99,75 \mathrm{aA}$ \\
\hline 60 & $78,75 \mathrm{aC}$ & $95,75 \mathrm{aA}$ & $95,75 \mathrm{aA}$ & $95,25 \mathrm{bB}$ \\
\hline 120 & $79,00 \mathrm{aB}$ & $85,25 \mathrm{bA}$ & $77,25 \mathrm{cB}$ & $36,75 \mathrm{dC}$ \\
\hline $\mathrm{CV}(\%)$ & 0,27 & 4,32 & 7,21 & 9,81 \\
\hline
\end{tabular}

(Continua) 
Tabela 1 - Período de exposição e concentração de $\mathrm{GA}_{3}$ na Germinabilidade (G\%), Índice de Velocidade de Germinação (IVG) e Tempo Médio de Germinação (TMG) em sementes de Phaseolusvulgaris cv. "Ouro Vermelho".

(Conclusão)

\section{IVG}

$\begin{array}{ccccc}\mathbf{3 0} & 0,26 \mathrm{bB} & 0,28 \mathrm{bB} & 0,31 \mathrm{bA} & 0,27 \mathrm{bC} \\ \mathbf{6 0} & 0,31 \mathrm{bA} & 0,27 \mathrm{bC} & 0,31 \mathrm{bB} & 0,86 \mathrm{aB} \\ \mathbf{1 2 0} & 0,28 \mathrm{~dB} & 0,38 \mathrm{aC} & 0,41 \mathrm{aB} & 1,41 \mathrm{aA} \\ & & & & \\ \mathbf{C V}(\%) & 2,14 & 3,42 & 3,68 & 8,93\end{array}$

\section{TMG}

$\begin{array}{ccccc}30 & 3,82 \mathrm{aA} & 3,55 \mathrm{bB} & 3,23 \mathrm{aD} & 3,61 \mathrm{bB} \\ \mathbf{6 0} & 3,21 \mathrm{cB} & 3,71 \mathrm{aA} & 3,27 \mathrm{aB} & 1,17 \mathrm{cB} \\ \mathbf{1 2 0} & 3,47 \mathrm{bA} & 2,57 \mathrm{cB} & 2,42 \mathrm{bC} & 0,84 \mathrm{dC}\end{array}$

$\begin{array}{lllll}\mathbf{C V}(\%) & 1,87 & 3,56 & 4,25 & 6,11\end{array}$

1 Resultados seguidos por letras maiúsculas iguais na mesma coluna e minúsculas na mesma linha, não diferem entre si pelo teste de Tukey a $5 \%$.

$\mathrm{CV}(\%)=$ Coeficiente de Variação.

Resultados semelhantes aos obtidos neste trabalho foram observados em estudos com sementes de Hordeum vulgare (Poaceae), Bauhinia monandra Britt. (Fabaceae), Bauhinia ungulata L. (A). (Fabaceae), Lafoensia pacari A. St. Hil. (Lythraceae), Ormosia arborea (Fabaceae) e Tabebuia impetiginosa (Bignoniaceae) em que a germinabilidade (G\%) foi incrementada com o aumento da concentração de $\mathrm{GA}_{3}$ (SCHUURINK; SEDEE ; WANG, 1992; CURIEL ; MORAES, 2011; ALVES et al., 2012; BATISTA et $a l .$, 2012; FERNANDES et al., 2012) e o índice de velocidade de germinação (IVG) apresentou melhores resultados com a crescente concentração de ácido giberélico fornecido e aumento dos períodos de exposição às sementes (CURIEL ; MORAES, 2011; ALVES et al., 2012; BATISTA et al., 2012; FERNANDES et al., 2012). Elevadas concentrações de ácido giberélico tendem a não só incrementar a G\%, mas também conferem ao processo germinativo maior resistência ao estresse hídrico (NI ; BRADFORD, 1993), pois tal fenômeno se relaciona ao aumento da secreção de $\alpha$-amilase na camada de aleurona, sintetizada em resposta ao $\mathrm{GA}_{3}$, e que promove a conversão do amido em glicose, que é usada no crescimento da plântula em desenvolvimento (BUCKERIDG SANTOS ; TINÉ, 2000; CURIEL ; MORAES, 2011; VERDOLIN-BENEDITO; CURIEL ; PEDROSO-DE-MORAES, 2012). Ainda, no ínterim do processo germinativo, o polissacarídeo manano, que está presente em inúmeras espécies, principalmente, em sementes de Fabaceae, não apresenta apenas a função de reserva, pois, este também confere grande dureza àquelas que os acumulam e isso é associado a um sistema de proteção embrionário contra danos mecânicos. Assim, a degradação de tal carboidrato pode ser induzida por ácido giberélico, estimulando a germinação (BUCKERIDG; SANTOS ; TINÉ, 2000).

Dessa forma, a presença de giberelinas sintéticas, estimula quantitativamente a G\% e a velocidade de germinação (NI ; BRADFORD, 1993; VERDOLIN-BENEDITO CURIEL ; PEDROSO-DEMORAES, 2012). Ainda, com relação ao IVG, tal afirmação é corroborada pelos resultados encontrados 
neste trabalho e aos observados para Annona squamosa (Annonaceae), para a qual, maiores concentrações de $\mathrm{GA}_{3}$ utilizadas, de 50 a $200 \mathrm{mg} \cdot \mathrm{L}^{-1}$, apresentaram maiores índices para a velocidade de germinação (FERREIRA; ERIG ; MORO,2002).

Incrementos em relação ao IVG também foram obtidos para Annona cherimola (Annonaceae) quando submetidas a elevadas concentrações do regulador vegetal (JUBES et al.,1975). Contudo, análises de germinação realizadas com Guarea guidonia (L.) Sleum (Meliaceae), para determinação da influência de $\mathrm{GA}_{3}$ na germinação das sementes em três concentrações $\left(300,400\right.$ e $\left.600 \mathrm{mg} \cdot \mathrm{L}^{-1}\right)$, não demonstraram diferenças significativas com relação ao IVG (CASTRO et al., 1999).

O tempo médio de germinação (TMG) e importante para se avaliar a rapidez de ocupação de uma espécie em uma comunidade (FERREIRA et al., 2001). Neste trabalho, para tal variável, a exposição a diferentes concentrações de $\mathrm{GA}_{3}$ apresentaram melhor valor médio em relação à exposição das sementes por 30 minutos em água (Tabela1) sendo que as crescentes concentrações de $\mathrm{GA}_{3}$ utilizadas nas sementes de Phaseolus vulgaris cv. "Ouro Vermelho" influenciaram negativamente tal variável. Corroborando tais resultados, podemos citar os obtidos para sementes de Actinidia chinesis Planc. (Actinidiaceae), as quais, não apresentaram resultados positivos quando tratadas com três concentrações distintas de $\mathrm{GA}_{3}(50,100$, $\left.150 \mathrm{mg} . \mathrm{L}^{-1}\right)$. Neste experimento, a maior concentração utilizada aumentou em até seis dias o TMG em comparação ao tratamento controle (YNOUE; ONO ; MARCHI, 1999). Fenômeno semelhante também foi constatado para L. pacari, para a qual, as sementes expostas à maior concentração de $\mathrm{GA}_{3}\left(20 \mathrm{mg} \cdot \mathrm{L}^{-1}\right)$ levaram 11 dias à mais para germinar dos que as submetidas às menores concentrações do regulador vegetal (FERNANDES et al., 2012).

\section{CONCLUSÃO}

Os resultados obtidos para as diferentes concentrações de $\mathrm{GA}_{3}$ e períodos de exposição foram negativos para Tempo Médio de Germinação e positivos para a Germinabilidade e Índice de Velocidade de Germinação, com estas últimas alcançando valores médios maiores à concentração de $20 \mathrm{mg} . \mathrm{L}^{-1}$ sob períodos de exposição ao regulador vegetal de 30 e 120 minutos respectivamente.

\section{REFERÊNCIAS}

ALVES, M.C.S.et al. Superação da dormência em sementes de Bauhinia monandra Britt. e Bauhinia ungulataL. - Caesalpinoideae. Revista Brasileira de Sementes, v. 22, p. 139-144,2000.

AYRES, M.et al. BioEstat: Aplicações estatísticas nas áreas das ciências biológicas e médicas. 5. ed. Belém: Sociedade Civil Mamirauá; Brasília: CNPq, 2007. 321 p.

BATISTA, R.O. et al. Morfometria carposeminal e germinação de Tabebuia impetiginosa (Mart.) Standl. pré-embebida em diferentes períodos de exposição e concentrações de $\mathrm{GA}_{3}$. Iheringia, Série Botânica, v. 67, p.237-243, 2012.

BUCKERIDGE, M. S.; SANTOS, H. P. ; TINÉ, M. A. S. Mobilization of storage cell wall polysaccharides in seeds. Plant Physiology and Biochemistry, v. 38, p.141-156, 2000.

CASTRO, E.M.et al. Influência do ácido giberélico e do nitrato de potássio na germinação de Guarea guidonia (L.) Sleum. Revista Árvore, v. 23, p.255-258,1999. 
CASTRO, P.R.C ; VIEIRA, E.L. Aplicação de reguladores vegetais na agricultura tropical. Guaíba: Agropecuária, 2001. 132p.

CIPOLLINI JR., D.F. Gibberellic acid treatment reduces the tolerance of field-grown common bean to leaf removal. Journal of Plant Growth Regulation, v. 16, p. 123-127, 1997.

CURIEL, A.C. ; MORAES, C.P. Germinação de Ormosia arborea (Vell.) Harms submetida a diferentes períodos de exposição e concentração de $\mathrm{GA}_{3}$ pós escarificação mecânica. Scientia Plena, v.7, p.1-6 , 2011.

EMBRAPA. Agência de Informação Feijão. Disponível em: <http://www.cnpaf. embrapa.br/>. Acesso em: 28 de fev. 2013.

FERNANDES, M.R.et al. Morfobiometria carpo seminal e germinação de Lafoensia pacari A. St. Hil. (Lythraceae) exposta a diferentes concentrações de $\mathrm{GA}_{3}$. Semina: Ciências Agrárias, v. 33(suplemento 1), p. 2571-2584,2012.

FERREIRA, G.; ERIG, P. R. ; MORO, E. Uso de ácido giberélico em sementes de fruta-do-conde (Annona squamosa $\mathrm{L}$.) visando à produção de musas em diferentes embalagens. Revista Brasileira de Fruticultura, v. 24, p. 178-182,2002.

JUBES, J.T.; MARTINEZ, H.; PADILLA, E. ; OSTE, C.A. Efectos de escarificacion, medio, posicion de siembra y acido gibberellico, sobre la germinacion de semillas en chirimoya (Annona cherimolia Mill). Revista Agronomica del Noroeste Argentino, v.12, p. 161-171, 1975.

KIGEL, J. ; GALILI, G. Seed development and germination.2. ed. New York: Plenum, 1995. 853p.

MORRIS, D. A. ; ARTHUR, E. D. Effects of gibberellic acid on patterns of carbohydrate distribution and acid invertase activity in Phaseolus vulgaris. Physiologia Plantarum,v. 65, p. 257-262, 1985.

NI, B. R. ; BRADFORD, K.J. Germination and dormancy ofabscisic acid and gibberellin - deficient mutant tomato (Lycopersicum esculentum) seeds. Plant Physiology, v. 101, p. 607-617,1993.

RAMALHO, M.A.P. Cultivo do Feijão da Primeira e Segunda Safras na Região Sul de Minas Gerais. Embrapa Arroz e Feijão: sistemas de produção, v. 6, p.1-2, 2005. Disponível em: <http://sistemasdeproducao.cnptia.embrapa.br/FontesHTML/Feijao/> Acesso em: 28 fev. 2013.

RAVEN, P. H.; EVERT, R. F. ; EICHHORN, S. E. Biologia Vegetal. 7. ed. Rio de Janeiro, RJ: Guanabara Koogan, 2007.521 p.

SALISBURY, F. B. ; ROSS, C. W. Plant Physiology. 4. ed. California: Wadsworth Publishing Company, $1992,682 \mathrm{p}$.

SCHUURINK, R.C.; SEDEE, N. J. A. ; WANG, M. Dormancy of the barley grain is correlated with gibberellic acid responsiveness of the isolated aleurone layer. Plant Physiology, v. 100, p. 1834-1839, 1992.

SKENE, K.G. M. Stimulation of germination of immature bean embryos by gibberellic acid. Planta, v. 87, p. 188-192,1969.

SOUZA, C.A.; COELHO, C.M.M.; AMARANTE, C.V.T.; MIQUELUTTI, D. J.; VIEIRA, S. P. ; ARCARO, T.F. Proposta para modificar a arquitetura de plantas de feijão pelo uso de ácido giberélico. Inter Science Place, v. 1, p. 1-14, 2009.

SOUZA, C.A.et al. Influência do ácido giberélico sobre a arquitetura de plantas de feijão no início de desenvolvimento. Acta Scientiarum. Agronomy, v. 32, p. 325-332.2010. 
SWARNKAR, S. K.; POONAM, S. ; MAURYA, C.L. Influence of plant growth regulators on aged seeds of soybean (Glycine max).Progressive Agriculture, v. 4, p. 66-68, 2004.

TAIZ, L. ; ZEIGER, E. Fisiologia Vegetal. 3. ed. Porto Alegre, RS: Artmed, 2004. 719 p.

VERDOLIN-BENEDITO, P.; CURIEL, A. L. ; PEDROSO-DE-MORAES, C. Influência do período de pré-embebição e de diferentes concentrações de ácido giberélico na germinação e crescimento de Mimosa caesalpiniaefolia Benth. Revista em Agronegócios e Meio Ambiente,v.5, p. 593-604, 2012.

VIEIRA, C.; PAULA-JÚNIOR, T. J. ; BORÉM, A. Feijão: aspectos gerais e cultura no Estado de Minas. Viçosa: UFV. 1998. 596 p.

VIEIRA, E. H. N. ; RAVA, C.A. Características botânicas e fisiológicas da semente. In: VIEIRA, E. H. N. ; RAVA, C.A. (Eds.). Semente de feijão: produção e tecnologia. Santo Antonio de Goiás: Embrapa Arroz e Feijão, 2000. Pp. 25-38.

YNOUE, C. K.; ONO, E. O. ; MARCHI, L.O.S. Efeito do $\mathrm{GA}_{3}$ na germinação de sementes de Kiwi (Actinidia chinensis Planch.). Scientia Agricola, v. 56, p. 9-12. 1999. 
